\title{
Full-thickness rectal prolapse following posterior vaginal repair: something to worry about?
}

\author{
F. Ross $・$ R. Dawson $\cdot$ J. Cooper
}

Received: 6 March 2012 / Accepted: 6 March 2012 / Published online: 12 April 2012

(C) The International Urogynecological Association 2012

Rectocoele is a common condition, which often leads to the need for surgical repair [1-3]. Common risks and complications of surgical repair discussed and documented on consent forms include: need for repeat repair; failure to improve symptoms; painful intercourse; unmasking of urinary incontinence and urinary retention; the usual surgical risks - thromboembolic events, bleeding, infection [4]. Following an interesting case, namely, a full-thickness rectal prolapse after a posterior trocar-inserted vaginal polypropylene mesh repair of a fourth-degree rectoenterocoele, we looked at the literature on this issue.

A literature search using the terms rectocoele, posterior repair, mesh repair of rectocoele, rectal prolapse, pelvic floor repair and concurrent rectal prolapse and rectocoele was undertaken. There were no reported cases that demonstrated a full-thickness rectal prolapse following mesh repair for rectocoele. There was a case reported by South and Amundsen in 2007 of an overt rectal prolapse following repair of a fourth-degree vault prolapse with colpocleisis and levator ani plication [5]. Our case would appear to be the first experienced postoperatively after performing an Avaulta mesh procedure. However, the aetiology of both is likely to be similar and is likely to be underreported.

Risk factors for rectocoele include high parity (especially large birth weights delivered vaginally), chronic raised intraabdominal pressure (chronic cough, persistent heavy lifting,

F. Ross $\cdot$ R. Dawson $\cdot$ J. Cooper

University Hospital of North Staffordshire,

Stoke on Trent ST4 6QG, UK

J. Cooper $(\bowtie)$

Department of Gynaecology,

University Hospital of North Staffordshire,

Stoke on Trent ST4 6QG, UK

e-mail: jasoncooper@doctors.org.uk constipation), age and increased body mass index (BMI) [2, 6]. Previous vaginal-wall repair also increases the risk of developing vaginal wall prolapse; it is often quoted that one in three women who undergo vaginal-wall repair will need further surgery, with some studies showing reoperation rates of $43 \%$ with standard repair techniques [7] and others objective failure rates at 1 year of $43 \%$, and objective failure rates of $40 \%$ and subjective failure of $24 \%$ at 5 years of follow-up $[8,9]$. Surgical treatment of a rectoenterocoele includes posterior colporrhaphy with or without mesh or an abdominal procedure such as a sacrocolpopexy. Our unit has had success with mesh repair [10], as measured using the International Consultation on Incontinence Modular Questionnaire-Vaginal Symptoms (ICIQ-VS) questionnaire, and hence vaginal mesh was used in the case that we were involved in.

A full-thickness rectal prolapse occurs when all layers of the rectal wall protrude through the anus [11]. This most commonly affects elderly women with a history of obstetric trauma causing weakening of the pelvic floor and damage to the pudendal nerve [11]. This then leads to weakness of the anal sphincters, predisposing to rectal prolapse. Other risk factors for rectal prolapse include obesity, increasing age and chronically raised intra-abdominal pressure - a significant overlap with the risk factors for pelvic organ prolapse (POP) [12]. It is therefore not surprising that the two defects commonly coexist [12].

It is accepted that connective tissue damage and disorders predispose to POP. The more significant the prolapse, the more likely it is that the damage is not confined to one area but distributed throughout the pelvis and that repairing or supporting one defect may shift the balance in the pelvis and thus expose or provoke a defect in another area. South and Amundsen hypothesised that repair of the defect in the posterior vaginal wall displaced the rectum out of the 
vagina, which then took the line of least resistance - through the anus [5]. We presume the rectum is put under stretch into the rectocoele, masking any true rectal problem. Once the vaginal wall is correctly positioned, the line of least resistance would be through the anus. Much like masking of stress urinary incontinence with a cystocoele, a rectal defect may have been masked by our patient's vaginal defect.

It has been suggested that women presenting with POP should be examined and assessed for evidence of rectal disorders (and vice versa), as it is likely that the number of women with concurrent genital and rectal prolapse is higher than reported [12]. Should both defects be apparent preoperatively, then the risk to patients may be decreased if gynaecological and colorectal procedures are undertaken at the same time, as many of these patients are elderly with multiple medical comorbidities, which increases their surgical risk [12]. This would require specific questions regarding rectal prolapse to be asked to ensure that nothing was missed. This would then allow a decision regarding more formal investigation for evidence of occult rectal prolapse.

It is likely that concomitant POP and rectal prolapse are more common than reported due to similarities in the risk factors for both. Investigation for each should be considered at presentation with one, but it has not been formally investigated as to whether this would be a viable option with regards to patient acceptability, cost-effectiveness and riskbenefit analysis [12]. However, in both our case and that of South and Amundsen the patients had normal colorectal examinations and still developed rectal prolapse as a postoperative complication of pelvic organ repair. It also has not been determined whether mesh repair increases support to the posterior compartment significantly enough to increase the risk of rectal prolapse compared with repair without mesh. However, we do know that 3 years after posterior vaginal mesh repair, $74 \%$ of patients who had symptoms of obstructive defaecation preoperatively had no such symptoms following surgery [10]. This indicates the significant effect that posterior vaginal defect repair can have on rectal function. Indeed, surgery for POP can sometimes improve rectal prolapse or make it worse, although it is most likely to have little effect. At present, it is seems best to continue to assess patients through routine history and examination, and if any rectal abnormalities are indicated, then discussion at a formal multidisciplinary team meeting is appropriate. Urogynaecologists should be aware of the association of concomitant rectal and vaginal prolapse and that both can be treated surgically at the same time. Patients should be carefully asked about previous rectal symptoms and warned that there will be a small chance of unmasking or creating rectal prolapse with posterior vaginal repair surgery. Therefore, regarding the consent process for rectocoele repair, perhaps we now need to add unmasking or development of new rectal prolapse as a complication of posterior compartment vaginal surgery.

Conflicts of interest None.

\section{References}

1. Monga A (2004) Management of the posterior compartment. Br J Obstet Gynaecol 111:73-78

2. Hendrix SL, Clark A, Nygaard I, Barnabei V, McTiernan A (2002) Pelvic organ prolapse in the Women's Health Initiative: gravity and gravidity. Am J Obstet Gynecol 186:1160-1166

3. Olsen AL, Smith VJ, Bergstrom JO, Colling JC, Clark AL (1997) Epidemiology of surgically managed pelvic organ prolapse and urinary incontinence. Obstet Gynecol 89:501-506

4. Royal College of Obstetricians \& Gynaecologists: Consent Advice No: 5 (2009) Vaginal Surgery for Prolapse

5. South M, Amundsen C (2006) Overt rectal prolapse following repair of stage IV vaginal vault prolapse. Int Urogynecol J 18:471-473

6. Cooper J (2011) Pelvic Floor Surgery. In: Arulkumaran S, Regan L, Papageorghiou A, Monga M \& Farquharson D (eds.) Oxford Desk Reference: Obstetrics \& Gynaecology. Oxford University Press, Oxford, pp. 710-11.

7. Sand PK, Koduri S, Lobel RW, Winkler HA, Tomezsko J, Culligan PJ, Goldberg R (2001) Prospective randomized trial of polyglactin 910 mesh to prevent recurrence of cystoceles and rectoceles. Am J Obstet Gynecol 184:1357-1362

8. Carey M, Higgs P, Goh J, Lim J, Leong A, Krause H, Cornish A (2009) Vaginal repair with mesh versus colporrhaphy for prolapse: a randomised controlled trial. Br J Obstet Gynaecol 116:13801386

9. Miedel A, Tegerstedt G, Mörlin B, Hammarström M (2008) A 5year prospective follow-up study of vaginal surgery for pelvic organ prolapse. Int Urogynecol J 19:1593-1601

10. Bondili A, Deguara C, Cooper J (2012) Medium term effects of a monofilament Polypropylene mesh for pelvic organ prolapse and sexual function symptoms. J Obstet Gynaecol 32(3):285-290

11. Felt-Bersma RJF, Stella MTE, Cuesta MA (2008) Rectal prolapse, rectal intussusception, rectocele, solitary rectal ulcer syndrome and enterocele. Gastroenterol Clin North Am 37:645-648

12. Dekel A, Rabinerson D, Ben RZ, Kaplan B, Mislovaty B, Bayer Y (2000) Concurrent genital and rectal prolapse: two pathologies - one joint operation. Br J Obstet Gynaecol 107:125-129 